\section{Biochirurgie in der Behandlung chronischer Wunden}

Zusammenfassung. Chronische Wunden stellen eine Herausforderung für das heutige Gesundheitswesen dar. Ein Grundprinzip der Behandlung ist das Debridement zur Entfernung devitalen Gewebes zur Verhinderung von Wundinfektionen und zur Heilungsförderung. Die Biochirurgie (syn. Maden- oder Larventherapie) stellt eine aussichtsreiche Ergänzung zum Spektrum der bekannten chirurgischen und topischen konservativen Behandlungsmaßnahmen dar. Die vorliegende Arbeit gibt einen kurzen Überblick zur Geschichte, Entomologie, Biochemie und medizinischen Indikationen der Biochirurgie und des praktischen Einsatzes der Larven.

Biosurgery for the Treatment of Chronic Lesions. Chronic wounds are a challenge of modern health care. A basic principle of treatment is the debridement to remove devitalized tissue to prevent wound infection and support healing. Biosurgery (syn. maggot or larval therapy) is a promising adjunct to the whole spectrum of surgical and topical treatment methods. The present paper gives a brief review on history, entomology, biochemistry and medical indications of biosurgery and the practical handling of maggots.

\section{Geschichte}

Es war kein geringerer als Ambrose Paré [1], der die vorteilhaften Wirkungen einer Myasis bei eitrigen Kriegswunden auf dem Schlachtfeld von St. Quentin dokumentiert hat [2,3]. Während des Syrienfeldzuges 1829 beobachtete Baron D. J. Larrey in Napoleons Armee, dass Fliegenlarven die Granulation bei Kriegswunden fördern und die Abheilungszeit verkürzen können [4]. Zum ersten klinischen Gebrauch der Larven kam es entgegen der geltenden militärischen Vorschriften während des Amerikanischen Bürgerkrieges durch J. F. Zacharias, einem Chirurgen der Konföderationsarmee, und Dr. J. Jones, einem Offizier des medizinischen Dienstes [5]. Im 1. Weltkrieg beschrieb William Bear die Wundreinigung durch Fliegenlarven bei zwei verwundeten Soldaten. Er ist es auch, der die wissenschaftliche Bearbeitung der Larven als Antiseptika initiierte

Akt Dermatol 2001; 27: 183-186

(c) Georg Thieme Verlag Stuttgart · New York ISSN 0340-2541
U. Wollina, Kerstin Karte, Claudia Herold, Annette Looks

Klinik für Dermatologie und Allergologie am Klinikum der Friedrich-Schiller-Universität Jena (Direktor: Prof. Dr. med. P. Elsner)

[6]. Stanton K. Livingston, ein Student von Baer, entwickelte nicht allein technische Hilfsmittel zur Larvenzucht, sondern stellte auch die Hypothese des „active principle“ auf, welches er aus Extrakten dieser Tiere gewann [7,8]. Während der 30er Jahre erlebte die Biochirurgie eine Blüte und wurde $\mathrm{zu}$ einer populären Behandlungsmethode in Nordamerika und Europa $[9,10]$. Das änderte sich schlagartig mit Einführung der Antibiotika.

Heute stehen wir dem Problem multiresistenter Keime gegenüber und die Biochirurgie wird für uns wieder attraktiver.

\section{Die Fliegen}

Es wurden verschiedene Spezies geprüft. Es eignen sich Fliegen der Gattung Calliphorina. Sie müssen ausschließliche Nekrophagen sein, d.h. sich nur vom devitalen Gewebe ernähren. Heute wird Lucilia sericata für die Biochirurgie gezüchtet. Die Larven schlüpfen nach $12-24 \mathrm{~h}$ aus den Eiern. Sie sind dann 1$2 \mathrm{~mm}$ groß und sehr mobil. Zirka 200 Larven verdauen bis zu $15 \mathrm{~g}$ nekrotischen Gewebes pro Tag und wachsen rasch heran [11]. Nach 4-5 Tagen erreichen sie die Reife und durchlaufen eine Metamorphose [12].

\section{Wichtige Schritte auf dem Weg zur Biochirurgie}

Der erste kritische Schritt ist die Züchtung geeigneter Larven in ausreichender Menge. Dabei muss eine Kontamination der Eier und Larven mit Bakterien und/oder Pilzen vermieden werden [13 - 15]. Heute sind steril aufgezogene Larven verfügbar [16].

Die Analyse der aktiven Inhaltsstoffe des Larvensekretes ist ein weiterer wichtiger Schritt. Bereits Livingston beschrieb die folgenden Substanzen: Sulfhydrylradikale, Allantoin, Calcium, Cystein, Gluthation und „embryonic growth stimulating substances“ [7] (Tab.1). Prete (1997) konnte kürzlich zeigen, dass die Hämolymphe und Verdauungssekrete wachstums-stimulierend für humane Fibroblasten sind. Sie wirken ko-stimulatorisch zu EGF- und Interleukin-6 [17]. Klinische Beobachtungen unterstützen diese Befunde. Auch hier scheinen Larvensekrete nicht allein das Debridement sondern auch die Wundheilung zu fördern [18]. 
Tab. 1 Inhaltsstoffe der Larvensekrete

Allantoin
Harnstoff
Calciumcarbonat
Enzyme wie
Trypsin
Chymotrypsin-artige Enzyme (LCTa, LCTb)
Leucinaminopeptidase
Carboxypeptidase A \& B
Serinproteasen

\section{Wie verwendet man Larven?}

Die praktische Anwendung der Biochirurgie bringt 3 Hauptprobleme mit sich:

- Die mobilen Larven sollen in der Wunde verbleiben.

- Sie benötigen ausreichend Sauerstoff.

- Die Wunde muss feucht gehalten werden, aber nicht überfeuchtet.

Zur Wundabdeckung nach Larvenapplikation werden Chiffonnetze in Kombination mit Hydrokolloiden eingesetzt [16,2022]. Wir haben Vacusealgel (Coloplast ${ }^{\circledR}$ ) mit dem „larval therapy system“ (Surgical Materials Testing Laboratory, Bridgend, UK) kombiniert. Als Sekundärverband kommt Oprasorb ${ }^{\circledR}$ (Lohmann) zum Einsatz, um überschüssiges Exsudat aufzunehmen. Laminatverbände mit genügender Sauerstoff-Permeabilität sind ebenfalls geeignet (Tielle ${ }^{\circledR}$, Johnson and Johnson). Nach 3 bis 5 Tagen werden die Larven mit Wasser abgespült. Die Behandlung kann wiederholt vorgenommen werden, falls das Debridement nicht ausreichend war. Die so vorbereiteten Wunden eignen sich auch zur Haut-Transplantation.

\section{Medizinische Indikationen}

Die Hauptindikation stellt die nekrotische, infizierte oder infekt-gefährdete Wunde dar. Die Wunde darf jedoch nicht ausgetrocknet sein. Aufgrund der Sauerstoffabhängigkeit der Larven sollten die Wunden eine großflächige Öffnung nach außen besitzen. Fistelgänge sind nicht geeignet. Ein Einsatz in der Nähe von Körperöffnungen ist u.U. problematisch, wenn es um die Entfernung der Tiere geht.

In einer klinischen Untersuchung von Weil et al. [23] über 56 Patienten mit Osteomyelitis, Weichteilinfektionen und malignen Ulzera wird über hervorragende Ergebnisse berichtet. Andere Autoren bestätigen die Therapieerfolge [9, 24-26]. Robinson [9] hat über 5750 Patienten berichtet, die bei 605 Ärzten/ Krankenhäusern in den USA und Canada behandelt wurden. 91,2\% der Ärzte waren mit der Biochirurgie zufrieden.

Sherman et al. [27] haben jüngst eine prospektiv kontrollierte Studie bei querschnittsgelähmten Patienten und Dekubitalulzera durchgeführt. Die Biochirurgie führte zu einer durchschnittlichen Verkleinerung der Wundfläche um $22 \%$ pro Woche während die Patienten ohne Biochirurgie eine durchschnittliche Zunahme der Wundfläche um 21,8\% pro Woche zeigten $(p<0,001)$. Nach unserer Erfahrung eignen sich diabetische Ulzera und Dekubitalulzera ausgezeichnet für die Lar-
Tab. 2 Indikationen zur Biochirurgie (Auswahl)

Osteomyelitis

(Baer 1931; Livingston \& Price 1940; Sherman \& Pechter 1988)

Mastoiditis

(Robinson 1935; Horn et al. 1976)

Karbunkel

(Ferguson \& McLaughlin 1935)

Nekrotische gangränöse Wunden

(Robinson 1935; Thomas et al. 1996, 1997)

Diabetisches Fußulkus

(Robinson 1935; Mumcuoglu et al. 1998)

Dekubitus

(Sherman et al. 1995; Thomas et al. 1996, 1997)

Pyoderma gangraenosum

(Boon et al. 1996)

Maligne Wunden

(Weil et al. 1933; Bunkis et al. 1985; Reames et al. 1988)

ventherapie. Aber auch Patienten mit gemischten arteriovenösen und posttraumatischen Ulzera sowie Ulzera bei Thrombangitis obliterans oder Pyoderma gangraenosum lassen sich sehr gut biochirurgisch reinigen. Eine kleine Übersicht zu den möglichen Indikationen gibt Tab. 2.

\section{Ausblick}

Das öffentliche Interesse an der Biochirurgie wächst. Die Methode ist nebenwirkungsarm und sicher. Sie ermöglicht eine maximale Erhaltung des vitalen Gewebes bei gleichzeitiger Anregung der Wundheilung. Mit einer weiteren Charakterisierung der Inhaltsstoffe der Larven sind neue Lokaltherapeutika in der Zukunft zu erwarten. Schon jetzt ist die Biochirurgie ein effektives Instrument in der Behandlung von Problemwunden (Abb. 1 u. 2).

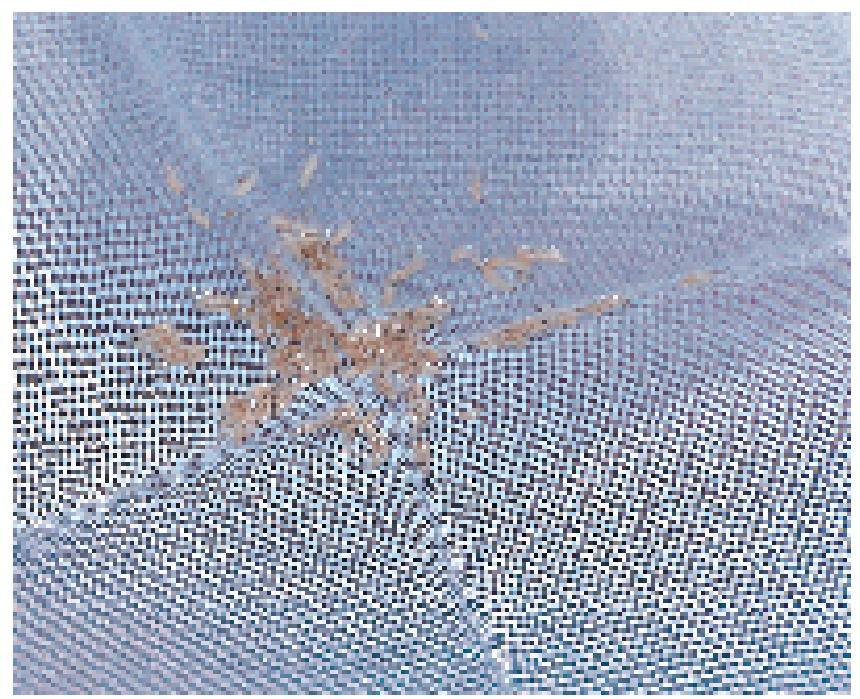

Abb. 1 Frisch geschlüpfte Fliegenlarven für die Biochirurgie. 
a

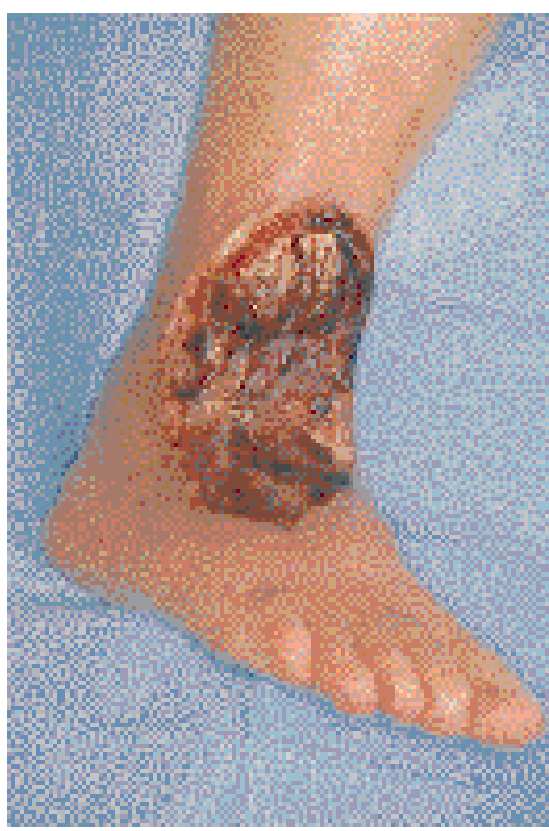

b

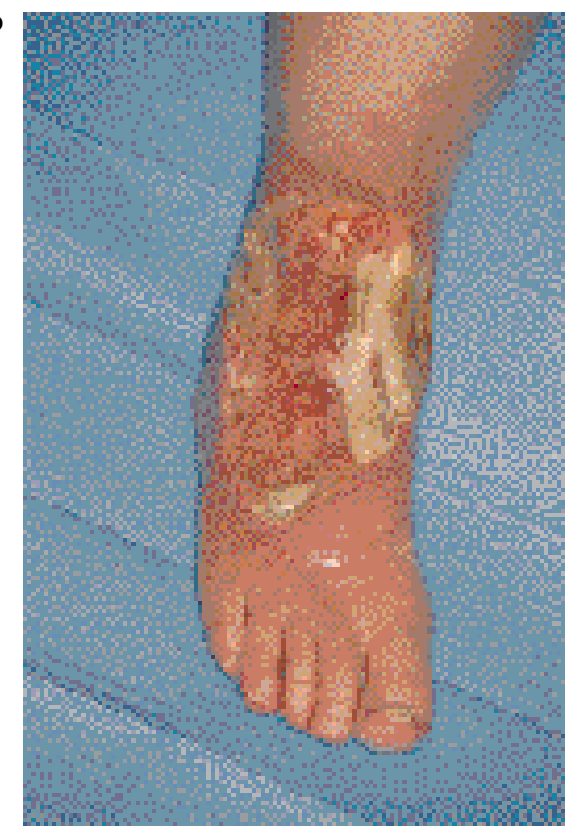

Abb. 2 a Therapieresistentes diabetisches Fußulkus vor Behandlung mit Belägen, Nekrosen und starker bakterieller Besiedlung. b Vier Tage nach Einsatz der Biochirurgie zeigt sich ein komplett gereinigtes Ulkus mit angeregter frischer Granulation und Wundflächenverkleinerung.

\section{Literatur}

${ }^{1}$ Paré A. The battle of S. Quentin (1557). In: Keynes G (Ed). The Apologie and Treatise of Ambroise Paré. Chicago: The University of Chicago Press, 1952: 68 - 70

2 Goldstein HI. Maggots in the treatment of wound and bone infections. J Bone Joint Surg 1931; 13: 476-478

${ }^{3}$ Gelbart M. Ancient and modern: the best of both. Nursing Times 1998; 94: 69-73

${ }^{4}$ Larrey DJ. Des vers ou larves de la mouche bleue. Clin Chir 1829; 1: 51

${ }^{5}$ Jones J. Confederate military prison hospital at Andersonville, Ga. Contributions relating to the causation and prevention of disease, and to camp diseases. In: Flint A (Ed). Sanitary memoirs (Medical Volume). New York: Hurd \& Houghton, 1867: 521
${ }^{6}$ Baer WS. The treatment of chronic osteomyelitis with the maggot (larvae of blowfly). J Bone Joint Surg 1931; 13: 428 - 475

${ }^{7}$ Livingston S K. The therapeutic active principle of maggots. J Bone Joint Surg 1936; 18: $751-756$

${ }^{8}$ Graner JL. S.K. Livingston and the maggot therapy of wounds. Military Med 1997; 162: 296 - 300

${ }^{9}$ Robinson W. Progress in maggot therapy in the United States and Canada in the treatment of suppurative diseases. Am J Surg 1935; 29: $67-71$

${ }^{10}$ Martini E. Über parasitische Fliegenlarven nebst Bemerkungen über die Kultivierbarkeit pathogener Mikroorganismen. Dermatol Wochenschr 1929; 15: 548 - 552

${ }^{11}$ Robinson W, Norwood VH. The role of surgical maggots in the disinfection of osteomyelitis and other infected wounds. J Bone Joint Surg 1933; 15: 409-412

${ }^{12}$ Crosskey RW, Lane RP. House flies, blowflies and other allies (Calyptrate diptera). In: Lane RP, Crosskey RW (Eds). Medical Insects and Arachnoids. London: Chapman and Hall, 1995

${ }^{13}$ Livingston SK. Maggots in the treatment of chronic osteomyelitis, infected wounds, and compound fractures. Surg Gynecol Obstet 1932; 54: $702-706$

${ }^{14}$ Livingston SK, Prince LH. The treatment of chronic osteomyelitis with special reference to the use of the maggot active principle. J Am Med Assoc 1932; 98: 1143 - 1149

${ }^{15}$ Fine A, Alexander H. Maggot therapy. Technique and clinical application. J Bone Joint Surg 1934; 16: 572 - 582

16 Thomas S, Jones M, Shutler S, Andrews A. All you need to know about maggots. Nursing Times 1996; 92: 63 - 76

17 Prete PE. Growth effects of Phaeniccia sericata larval extracts on fibroblasts: mechanism for wound healing by maggot therapy. Life Sci 1997; 60: 505- 510

18 Sherman RA, Pechter EA. Maggot therapy: a review of the therapeutic applications of fly larvae in human medicine, especially for treating osteomyelitis. Med Vet Entomol 1988; 2: 225 - 230

${ }^{19}$ Erdmann GR, Khalil SK. Isolation and identification of two antibacterial agents produced by a strain of Proteus mirabilis isolated from larvae of the screwworm. J Med Entomol 1986; 23: 208 211

${ }^{20}$ Alderman C. New success for old treatment. Nursing Standard 1996; 10: $26-27$

${ }^{21}$ Sherman RA. A new dressing design for use with maggot therapy. Plast Reconstr Surg 1997; 100: $451-456$

22 Thomas S, Andrews A. The effect of hydrogel dressings on maggot development. J Wound Care 1999; 8: 75-77

${ }^{23}$ Weil GC, Simon RJ, Sweadner WR. A biological, bacteriological and clinical study of larval or maggot therapy in the treatment of acute and chronic pyogenic infections. Am J Surg 1933; 19: $36-48$

${ }^{24}$ Ferguson LK, McLaughlin CW. Maggot therapy: a rapid method of removing necrotic tissue. Am J Surg 1935; 29: 72 - 84

${ }^{25}$ Thomas S, Jones M, Shutler S, Jones S. Using larvae in modern wound management. J Wound Care 1996; 5: 60-69

${ }^{26}$ Boon H, Freeman L, Unsworth J. Larvae may help debridement. Nursing Times 1996; 92: 76-80

27 Sherman RA, Wyle F, Vulpe M. Maggot therapy for treating pressure ulcers in spinal cord injury patients. J Spinal Cord Med 1995; 18: $71-74$

${ }^{28}$ Tellam RL, Eisemann CH, Pearson RD. Vaccination of sheep with purified serine proteases from the secretory and excretory material of Lucilia cuprina larvae. Int J Parasitol 1994; 24: 757-764

${ }^{29}$ Casu RE, Pearson RD, Jarmey JM, Cadogan LC, Riding GA, Tellam RL. Excretory/secretory chymotrypsin from Lucilia cuprina: purification, enzymatic specificity and amino acid sequence deduced from mRNA. Insect Mol Biol 1994; 3: 201 - 211

${ }^{30}$ Horn KL, Cobb Jr AH, Gates GA. Maggot therapy for subacute mastoiditis. Acta Otolaryngol 1976; 102: 377-379 
${ }^{31}$ Thomas S, Jones M, Andrews A. The use of fly larvae in the treatment of wounds. Nursing Standard 1997; 12: 54-59

32 Mumcuogly KY, Ingber A, Gilead L, Stessman J, Friedman R, et al. Maggot therapy for the treatment of diabetic foot ulcers. Diabetes Care 1998; 11: 2030-2031

${ }^{33}$ Bunkis MD, Gherini S, Walton R. Maggot therapy revisited. West J Med 1985; 142: $554-556$

${ }^{34}$ Reames MK, Christensen C, Luce EA. The use of maggots in wound debridement. Ann Plast Surg 1988; 21: 388 - 391
Prof. Dr. Uwe Wollina

Abteilung für Dermatologie

Krankenhaus Dresden-Friedrichstadt

Friedrichstraße 41

01067 Dresden 